\title{
Microelectrode Recording for Deep Brain Stimulation of the Subthalamic Nucleus in Patients with Advanced Parkinson's Disease: Advantage or Loss of Time?
}

\author{
Ersoy KOCABICAK ${ }^{1,3,4^{*}}$, Onur ALPTEKIN ${ }^{4 *}$, Dursun AYGUN² , Onur YILDIZ², Yasin TEMEL 3,4 \\ ${ }^{1}$ Ondokuz Mayis University, Faculty of Medicine, Department of Neurosurgery, Samsun, Turkey \\ ${ }^{2}$ Ondokuz Mayis University, Faculty of Medicine, Department of Neurology, Samsun, Turkey \\ ${ }^{3}$ Maastricht University Medical Center, Department of Neurosurgery, Maastricht, The Netherlands \\ ${ }^{4}$ Maastricht University Medical Center, Mental Health and Neuroscience (MHENS), Maastricht, The Netherlands \\ * These authors have contributed equally.
}

This study has been presented at the XX|st The European Society for Stereotactic and Functional Neurosurgery (ESSFN) Congress between 16 and 21 September 2014 at the Maastricht, Netherlands.

Corresponding authors: Onur ALPTEKIN, Ersoy KOCABICAK onur_alptekin@hotmail.com; ersoykocabicak@gmail.com

\section{ABSTRACT}

AIM: To investigate the effect of using microelectrode recording (MER) on the length of time required to carry out a deep brain stimulation (DBS) procedure of the subthalamic nucleus in patients with Parkinson's disease (PD).

MATERIAL and METHODS: The time required to include MER in the DBS operation was calculated for the first and second sides in 24 patients with PD. The number of microelectrodes used on each trajectory for the first and second sides, and the percentage of permanent electrodes implanted on each trajectory for the first and second sides, were quantified.

RESULTS: The average times taken to use MER were $23.4 \pm 6.2$ minutes, $17.4 \pm 6.5$ minutes, and $41.2 \pm 6.3$ minutes for the first side, second side and total procedure, respectively. In $75 \%$ of patients, the permanent electrode was implanted at the planned target site for the first side, and in $61 \%$ of patients for the second side.

CONCLUSION: MER extends the time required to carry out the DBS procedure. However, during surgery, it provides real-time information on the electrodes' neurophysiological locations and helps the surgical team choose an alternative target if the planned target does not produce satisfying results.

KEYWORDS: Microelectrode recording, Subthalamic nucleus, Deep brain stimulation, Parkinson's disease

ABBREVIATIONS: AP: Anteroposterior, CSF: Cerebrospinal Fluid, CT: Computerized Tomography, DBS: Deep Brain Stimulation, Hz: Hertz, ICH: Intracerebral Hemorrhage, LEDD: Leva-Dopa Drug Dosage, MER: Microelectrode Recording, min: Minute, mm: Millimeter, MR: Magnetic Resonance, MRI: Magnetic Resonance Imaging, SD: Standard Deviation, SNr: Substantia Nigra Pars Reticulata, STN: Subthalamic Nucleus, PD: Parkinson's Disease, UPDRS: Unified Parkinson's Disease Rating Scale, V: Volt

\section{INTRODUCTION}

$\mathrm{D}$ eep brain stimulation (DBS) of the subthalamic nucleus (STN) has become a well-accepted treatment for patients with advanced Parkinson's disease
(PD) $(7,11,18)$. It is well-known that the success of DBS depends on the accuracy in targeting the STN (27). Imaging techniques, stereotactic precision, and intraoperative microelectrode recording (MER) procedures help with precise
Ersoy KOCABICAK 이 : 0000-0003-3445-1734 Onur ALPTEKIN (1) : 0000-0001-7547-7231
Dursun AYGUN : (D) : 0000-0002-7079-6142 Onur YILDIZ (1) : 0000-0002-2796-8770
Yasin TEMEL (10) : 0000-0002-3589-5604 
targeting. Historically, the STN was not detectable using magnetic resonance imaging (MRI) for DBS; therefore, using intraoperative MER was essential (2). However, with advancements in MRI, particularly the development of T2weighed images, the STN can be observed directly. Using this improved technology, centers began to apply direct targeting methods and rejected MER. There are two main reasons why centers stopped using MER. First, there is an apparent risk of increased bleeding, although some reports claim the opposite $(9,17,19,20,23,24,26)$. Second, more time is required to carry out the operation. In this study, we investigated the latter in a consecutive series of patients with PD who underwent DBS of the STN.

\section{MATERIAL and METHODS}

This is a prospective cohort study. Twenty-four consecutive patients with PD were included. The demographic data of the patients are presented in Table I. All patients underwent DBS of the STN with MER at the Ondokuz Mayis University (Samsun, Turkey) DBS center and were followed for at least one year postoperatively. Twenty-three patients were operated bilaterally and one patient unilaterally. All patients received preoperative clinical assessments using the Unified Parkinson's Disease Rating Scale (UPDRS) parts I/II/III and IV, Schwab and England, and Hoehn \& Yahr scoring in the medication on and off states, and preoperative psychiatric assessments. Informed consent was requested and obtained from all patients, and the study was approved by the local medical ethical committee.This study was conducted in accordance with the Declaration of Helsinki.

\section{Surgical Procedure}

Before surgery and for each patient, a preoperative MRI scan was performed (Siemens, 1.5-Tesla MR Scanner, Erlangen, Germany), and the STN was defined by direct targeting on T2-weighted MR images. The trajectory was planned and the number of MER electrodes was determined by the presence of vessels seen with gadolinium on the T1-weighted MR images. On the morning of surgery, a Leksell $G$ frame was mounted, and stereotaxic computerized tomography (CT) was performed without contrast with a slice thickness of $1 \mathrm{~mm}$ (Aquillon 16 CT scanner, Toshiba, Tokyo, Japan).

In the next step, the MRI and CT images were fused and stereotactic coordinates were calculated on the work station (Framelink 5, Medtronic Inc. Minneapolis, USA).

The doses of anti-parkinsonian drugs had been reduced in the days before surgery and administration of the drugs was halted 12 hours before the operation. All surgeries were performed under local anesthesia. Following anesthesiologic preparation with dexmedetomidine hydrochloride, a precoronal burr hole was made on the contralateral side from the most severe PD symptoms. Before placement of the MER electrodes, the patient's blood pressure was checked. The burr hole was covered with cottonoids to prevent substantial cerebrospinal fluid (CSF) leakage.
MER was performed with polyamide-coated tungsten microelectrodes (Medtronic; microelectrode 291; $10 \mu \mathrm{m}$ width, impedance $1.1 \pm 0.4 \mathrm{M} \Omega$; measured at $220 \mathrm{~Hz}$ ) in $1 \mathrm{~mm}$ steps from $10 \mathrm{~mm}$ above the target for the first $5 \mathrm{~mm}$, then in 0.5 $\mathrm{mm}$ steps until the end of measured STN activity and the start of substantia nigra pars reticulata (SNr) activity. Signals were recorded with the Leadpoint system (Medtronic, Minneapolis, USA).

Before the test stimulation, which was performed with the same electrodes, patients were examined by the neurologist and baseline values for the cardinal symptoms (rigidity, hypokinesia, and tremor if present) were obtained. The microelectrode with the most typical STN pattern and the longest recordings in $\mathrm{mm}$ was always selected for the test stimulation. Intraoperative macrostimulation was performed, with a frequency of $130 \mathrm{~Hz}$ and a pulse width of $60 \mu \mathrm{s}$, in a step-wise fashion every $2 \mathrm{~mm}$ within the recorded STN electrophysiological borders. At each $0.5 \mathrm{~V}$ step, the stimulus intensity was increased until a desired clinical improvement

Table I: Patient Data Prior to the Operation and 1-Year Post Operation

\begin{tabular}{|c|c|c|}
\hline & $\begin{array}{c}\text { Preoperative } \\
\text { demographic } \\
\text { data of the } \\
\text { patients }\end{array}$ & $\begin{array}{c}\text { Patient } \\
\text { scores at the } \\
\text { end of one } \\
\text { year }\end{array}$ \\
\hline Age & $51.6 \pm 9.63$ & - \\
\hline Disease duration & $10.4 \pm 4.2$ & - \\
\hline LEDD & $1245 \pm 224.5$ & - \\
\hline UPDRS Total Med-off & $82.2 \pm 24.6$ & - \\
\hline UPDRS Total Med-on & $35.1 \pm 9.1$ & - \\
\hline Improvement (\%) & $57.2 \pm 13.8$ & - \\
\hline UPDRS III Med-off & $51.2 \pm 13.2$ & - \\
\hline UPDRS III Med-on & $17.3 \pm 6.1$ & - \\
\hline Improvement (\%) & $66.2 \pm 14.1$ & - \\
\hline H\&Y Med-off & $3.6 \pm 0.6$ & - \\
\hline H\&Y Med-on & $2.5 \pm 0.4$ & - \\
\hline Schwab England \% Med-off & $34 \pm 13.6$ & - \\
\hline Schwab England \% Med-on & $89 \pm 1.8$ & - \\
\hline Beck & $9.1 \pm 2.6$ & - \\
\hline MMSE & $28.6 \pm 0.7$ & - \\
\hline UPDRS III Stim-off Med-off & - & $53.1 \pm 14.4$ \\
\hline UPDRS III Stim-on Med-off & - & $24.3 \pm 15.1$ \\
\hline Improvement (\%) & - & $54.2 \pm 16.8$ \\
\hline LEDD postop & - & $535 \pm 184.5$ \\
\hline Improvement (\%) & - & $57 \pm 8.2$ \\
\hline
\end{tabular}


was obtained or a rise in side effects was evaluated by a neurologist. Detailed clinical parameters were scored using the UPDRS including: tremor (if present) and rigidityin all four extremities, finger taps, hand movements and/or handgrips, and leg agility. If there were positive clinical results with low stimulation parameters, and side effects were absent or only visible at higher amplitudes, the test electrode was removed and replaced with a permanent electrode (Model 3389; Medtronic, Minneapolis, USA). If no satisfactory effect was acquired during the test stimulation, the microelectrode that proved to be the second best option was chosen for clinical evaluation. This was, typically, the trajectory with the second longest STN activity. The permanent electrode position was verified using fluoroscopy and the remaining microelectrodes were removed. Finally, the permanent electrode was fixed in the burr hole with methyl methacrylate. The same procedure was performed for the contralateral side. After the permanent electrodes' procedures were evaluated and symptomatic bleeding or other structural complications were investigated with T1-and T2-weighted scans, internalization of the pulse generator (Activa PC; Medtronic, Minneapolis, USA) was performed under general anesthesia.

\section{Quantification of Time-Related Details of MER}

The following durations were recorded: the time between the first microelectrode placement as well as the testing of the effects and side effects with macro stimulation, the marking of the chosen trajectory with fluoroscopy, removal of the microelectrode and implantation of macroelectrode, checks on the trajectory with fluoroscopy, and removal of other microelectrodes.

When the calculated and planned trajectories cannot be chosen, a second trajectory is required. The multiple electrodes' advantage is the immediate availability of a second-best trajectory based on the recordings. This approach's advantage is a gain in time.

The average coordinates for the $X, Y$, and $Z$ axes and the average coronal and sagittal trajectory angles were noted for all patients. The number of microelectrodes used on each trajectory for the first and second sides, and the percentage use of the pre-planned trajectory for permanent electrode implantation on the first and second sides, were also calculated.

\section{Postoperative Clinical Follow up}

UPDRS part III assessments were scored in the stimulation off/medication off and stimulation on/medication off states. The change in leva-dopa drug dosage (LEDD) at one year postoperatively was evaluated. Early and late postoperative surgical, hardware and psychiatric side effects and complications were investigated.

\section{Statistical Analysis}

Data are presented as means \pm standard deviations (SD) and were analyzed using the Wilcoxon signed ranks test. The level of statistical significance was defined as $p<0.05$. All data were analyzed using the SPSS 15.0 software package (Version 15.0).

\section{- RESULTS}

The average functional coordinates for the patients who were operated on in the right and/or left sides are presented in Table II. The average coronal and sagittal trajectory angles are illustrated in Figure $1 \mathrm{~A}, \mathrm{~B}$. One hundred and fifty-nine microelectrodes were used (81 right side, 78 left side) in total for all patients. The average number of microelectrodes used for the right and left sides was $3.5 \pm 0.77$ and $3.4 \pm 0.87$,

Table II: Average Functional Coordinates for Right and Left STN

\begin{tabular}{lcc}
\hline & Right STN & Left STN \\
\hline$X$ & $12.32 \mathrm{~mm} \pm 0.76$ & $-12.24 \mathrm{~mm} \pm 1.04$ \\
\hline$Y$ & $-1.80 \mathrm{~mm} \pm 0.72$ & $-2.31 \mathrm{~mm} \pm 0.74$ \\
\hline$Z$ & $-3.99 \mathrm{~mm} \pm 0.44$ & $-4.17 \mathrm{~mm} \pm 0.39$ \\
\hline
\end{tabular}

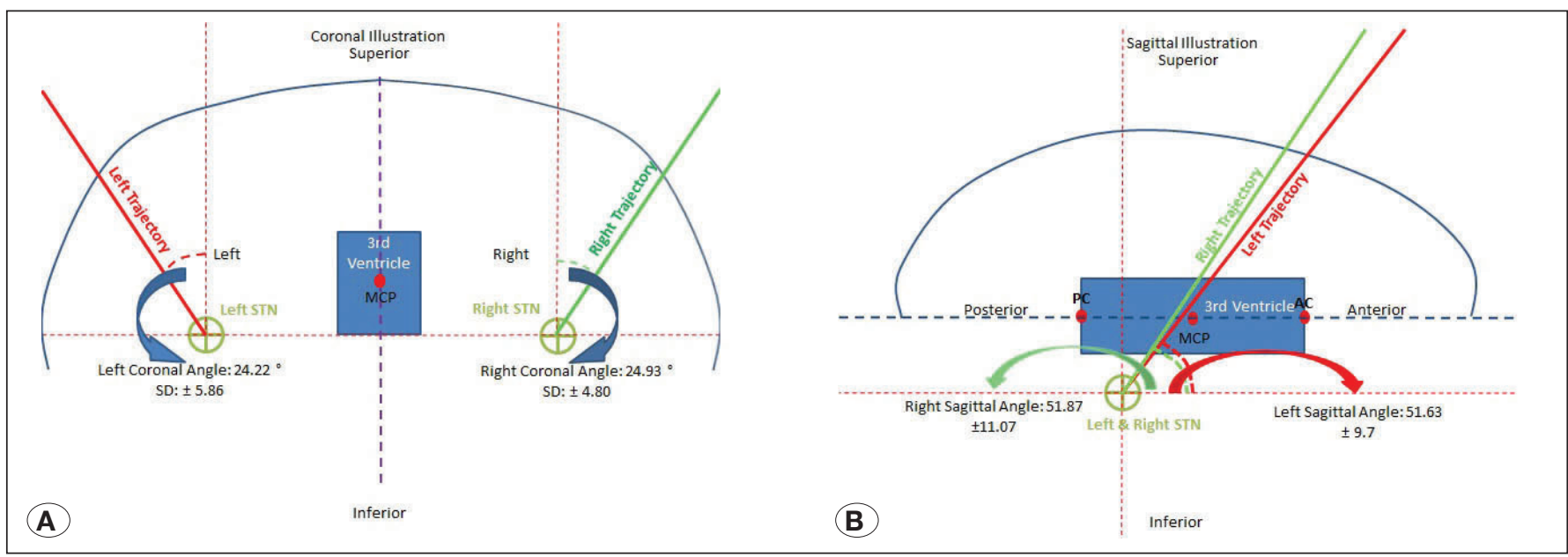

Figure 1: A) Coronal trajectory angles for the right and left STN trajectories depicted on a coronal illustration. B) Sagittal trajectory angles for the right and left STN trajectories depicted on a sagittal illustration. 
respectively. The average length of the STN measured with MER, for the first and the second sides to be operated on, were $5.6 \pm 1.8 \mathrm{~mm}$ and $4.8 \pm 1.5 \mathrm{~mm}$, respectively. Fortyseven permanent electrodes were implanted in all patients (first side: 24 , second side: 23 ). The first side to be operated on included placement of 9 right-side and 15 left-side permanent electrodes; the second side to be operated on included 15 right-side and 8 left-side permanent electrodes (Figure 2A-D). The time spent during the MER phase of the surgery for the first side, second side and total procedure was $23.4 \pm 6.2$ minutes, $17.4 \pm 6.5$ minutes, and $41.2 \pm 6.3$ minutes, respectively. UPDRS III scores, LEDD in Stim-On and Stim-Off states at the end of 1 year are presented in Table I. In this study, there were no intracerebral hemorrhages $(\mathrm{ICH})$ due to radiological assessments. Other intraoperative and postoperative complications, and the side effects related to surgery, hardware, and stimulation, are documented in Table III.

\section{DISCUSSION}

In this study, the average period of time spent during the MER phase of the surgery was $41.2 \pm 6.3$ min for bilateral DBS of the STN. Although there was no significant difference in the average number of microelectrodes used during MER on the first and second sides, there was $\sim 6$ minutes time difference between procedures on each side. In our experience, the level of cooperation from the patient decreases and confusion sometimes appears during MER of the second side. Accordingly, the surgeons attempt to finalize the MER of the second side as quickly as possible.
Table III: Complications and Side Effects Recorded One-Year Post Operation

\begin{tabular}{lcc}
\hline Complications/ & Intraoperative Postoperative \\
Side effects & $\mathbf{n}(\%)$ & $\mathbf{n}(\%)$ \\
\hline Intracranial Hemorrhage & - & - \\
\hline Seizure & - & - \\
\hline Mortality & - & - \\
\hline Local Infection & - & $1(4.1)$ \\
\hline Open Wound & - & $1(4.1)$ \\
\hline Presyncope / Syncope & $1(4.1)$ & - \\
\hline Gait Disorders & - & $3(12.5)$ \\
\hline Speech Disorders & $1(4.1)$ & $1(4.1)$ \\
\hline Weight Gain & - & $3(12.5)$ \\
\hline Hypomania & - & $2(8.3)$ \\
\hline Impulse Control Disorders & - & - \\
\hline Pshychotic Disorders & - & - \\
\hline Paresthesia & $3(12.5)$ & - \\
\hline Limitation of Eye Movements & $2(8.3)$ & - \\
\hline Apraxia of Lid Opening & $2(8.3)$ & - \\
\hline Facial Spasm & $1(4.1)$ & - \\
\hline
\end{tabular}

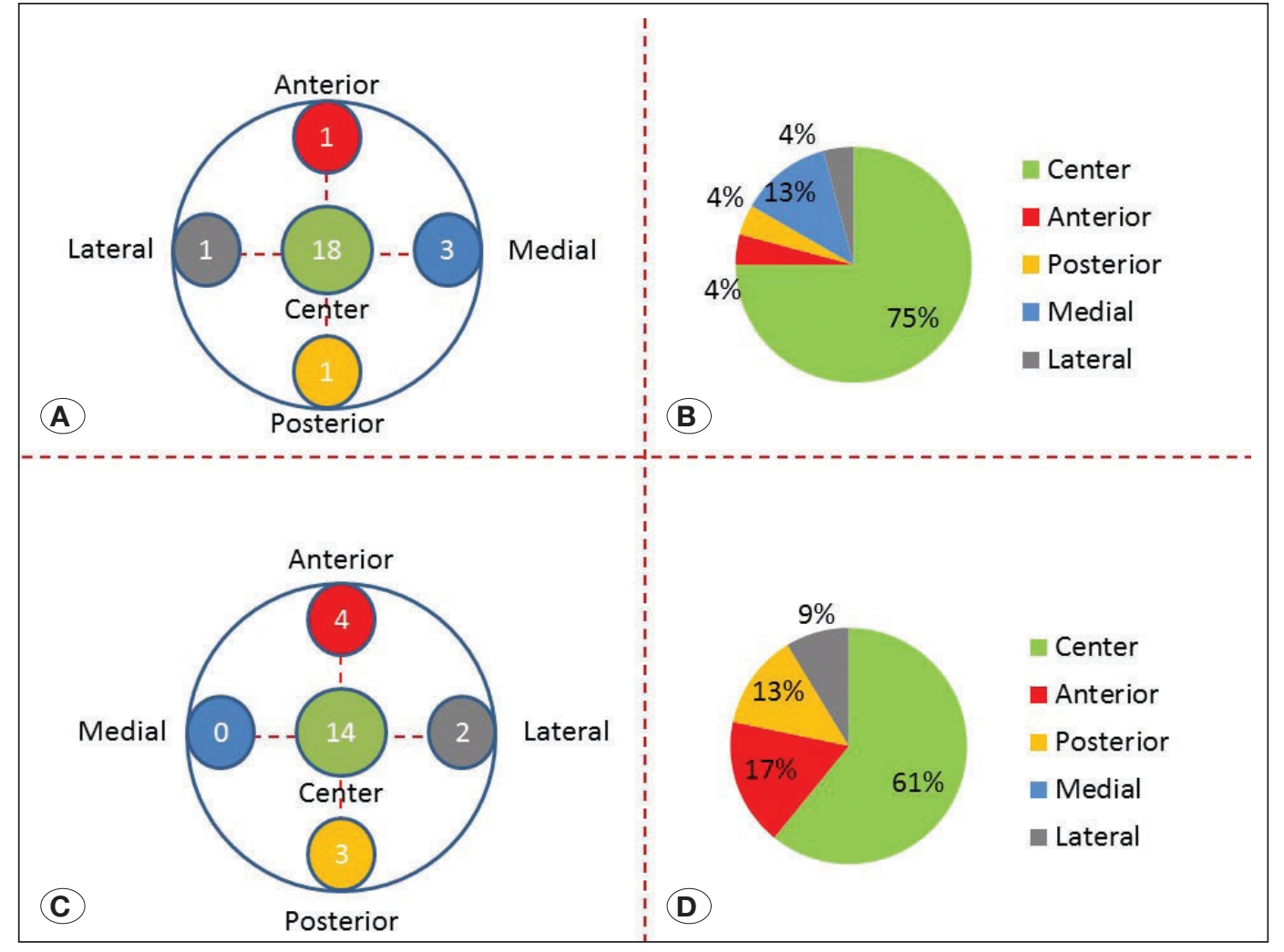

Figure 2: Illustrations of Ben's gun for the procedures on the first side and the second side (A and $\mathbf{C})$. The five circles of differing colors within Ben's gun demonstrate the five different trajectories. The numbers shared in these five trajectories denote the number of permanent electrode implantations for each trajectory in this series. The pie charts, shown in panels $\mathbf{B}$ and $\mathbf{D}$, indicate the percentage implantation for each trajectory in Ben's gun for the first side and the second side of the procedure, respectively. 
As far as we know, there has been no published work specifically measuring the time taken to perform MER during DBS surgeries to date. However, some studies have evaluated the durations of DBS procedures with and without MER $(6,17,22,25,30)$. Kinfe and Vesper reported that the average total DBS procedure times, calculated with and without MER, are $199 \mathrm{~min}$ in 36 patients and $201 \mathrm{~min}$ in 32 patients, respectively (12). Interestingly, the report indicates that the time taken for the procedure without MER is longer than when MER is performed.

In our experience, MER should start from $10 \mathrm{~mm}$ above the MRI-based target to $5 \mathrm{~mm}$ below it, performed in $1 \mathrm{~mm}$ steps for the first $5 \mathrm{~mm}$, followed by $0.5 \mathrm{~mm}$ steps until cessation of measurable STN activity. About 25 recordings are taken per side. Above the STN, irregular, low-frequency patterns from the anterior thalamus can be recorded, while non-specific pattern scan be found in the zona incerta. The STN shows typical electrophysiological activity, consisting of high-voltage spikes, burst firing of cells, and an obvious background expansion. MER is stopped when STN activity disappears and substantia nigra pars reticulata ( $\mathrm{SNr}$ ) activity, which consists of more regular cell firing patterns, appears. One explanation for the burst activity seen in the STN is the loss of dopaminergic input to the STN $(12,15,30)$. Dopaminergic neurons in the substantia nigra inhibit STN cells. Some publications suggest that the time taken to perform MER may be reduced by starting recordings at $6 \mathrm{~mm}$ instead of $10 \mathrm{~mm}$ from the MRIbased STN target (6). Although each recording takes 8-10 seconds, some authors claim even 1 second of MER per step is sufficient to define the STN's electrophysiological borders (22). To avoid orientation dysfunction in the patient, we prefer to start MER $10 \mathrm{~mm}$ above the target. We also believe that MER of the STN is an important tool for the identification of the border between the STN and SNr. Even with accurate AP and lateral coordinates, it is not easy to define the most inferior part of the STN if it is difficult to see the border between STN and SNr structures on T2-weighted images $(21,31)$.

The clinical outcome (improvement of UPDRS III scores) of our series, in which patients were operated on using the MER technique, is similar to results from studies using the same technique in the literature $(7,13,24)$. However, there are many studies performed without MER which have clinical results similar to those using MER $(1,29)$. Unfortunately, as far as we are aware, there have been no prospective randomized studies comparing clinical outcomes for patients who have undergone DBS operations with and without MER. Reck et al. published a series comparing the clinical outcomes of 32 patients with PD who underwent DBS of the STN, including 22 patients who were operated on with MER, and 10 patients without MER (19). They found a significantly better clinical outcome (UPDRS III scores) in patients undergoing MER compared with those who did not undergo MER.

In our study, there was no $\mathrm{ICH}$ occurrence, which is the most feared complication of DBS in the STN using MER. The estimated risk of an ICH in DBS of the STN varies between $0.2 \%$ and $5.6 \%$ in the literature $(1,4,24,28)$. Hariz claim that non-MER guided DBS surgery is five times safer in comparison to MER guided DBS surgeries in terms of $\mathrm{ICH}$ risk (10). Xiaowu et al. reported that increasing microelectrode trajectories seemed to increase the risk of $\mathrm{ICH}$, but no statistically significant difference was found (29). In our previous study, which included 220 patients with PD who underwent DBS of the STN, we found that the overall calculated risk of $\mathrm{ICH}$ was $1.81 \%$ per patient, $0.3 \%$ per recording electrode, and $0.23 \%$ per brain insertion (26). Other complications involved with performing DBS of the STN found in this study are similar to those reported in other publications $(3,5,8,16)$.

In this study, the percentage use of central trajectories for permanent electrode implantations in the first and second sides were $75 \%$ and $61 \%$, respectively. Although we used cottonoids to prevent brain shift due to CSF leakage, we believe that the percentage difference in use of the central trajectory for the first and second sides is related to this factor. Therefore, the MER of the STN may be much more important for electrode implantation on the second side. With the use of MER, error related to CSF leakage could be minimized or eliminated. Bour et al. reported that, in $50 \%$ of their cases, the central trajectory was used for permanent electrode implantation in a study of 29 PD patients when the authors performed DBS of the STN (6). In their series, they used a combination of direct and indirect methods on the work station for preoperative targeting. In another study, Reck et al. calculated the central trajectory implantation rate to be $73 \%$ in 22 patients with PD when DBS of the STN was performed (19). In our previous study, we reported a central trajectory implantation rate of $\sim 70 \%$ using a combination of direct and indirect targeting methods (14). Toda et al. reported that the implant rate of permanent DBS electrodes to the central trajectory was $81 \%$ in their series in which a $3 \mathrm{~T}$ MRI machine was used. Even the transition from 1.5T to 3.0T seems to improve targeting accuracy. A non-central trajectory is currently used for one in five patients (25).

\section{CONCLUSION}

Today, it is well-accepted that, through advancements in MRI techniques and technologies, it is feasible to perform the DBS procedure without using MER. However, we still believe that MER offers the DBS surgical team a choice of using a second trajectory, when needed, to improve the effect of the therapy and reduce the incidence of side effects.

\section{- REFERENCES}

1. Ben-Haim S, Asaad WF, Gale JT, Eskandar EN: Risk factors for hemorrhage during microelectrode-guided deep brain stimulation and the introduction of an improved microelectrode design. Neurosurgery 64(4):754-763, 2009

2. Benabid AL, Pollak P, Gao D, Hoffmann D, Limousin P, Gay E, Payen I, Benazzouz A: Chronic electrical stimulation of the ventralis intermedius nucleus of the thalamus as a treatment of movement disorders. J Neurosurg 84(2):203-214, 1996

3. Beric A, Kelly PJ, Rezai A, Sterio D, Mogilner A, Zonenshayn M, Kopell B: Complications of deep brain stimulation surgery. Stereotact. Funct Neurosurg 77(1-4):73-78, 2001 
4. Binder DK, Rau G, Starr PA: Hemorrhagic complications of microelectrode-guided deep brain stimulation. Stereotact Funct Neurosurg 80(1-4):28-31, 2003

5. Blomstedt P, Hariz M: Hardware-related complications of deep brain stimulation: A ten year experience. Acta Neurochir (Wien) 147(10):1061-1064, 2005

6. Bour LJ, Contarino MF, Foncke EM, de Bie RM, van den Munckhof P, Speelman JD, Schuurman PR: Long-term experience with intraoperative microrecording during DBS neurosurgery in STN and GPi. Acta Neurochir (Wien) 152(12):2069-2077, 2010

7. Deuschl G, Schade-Brittinger C, Krack P, Volkmann J, Schäfer H, Bötzel K, Daniels C, Deutschländer A, Dillmann U, Eisner W: A randomized trial of deep-brain stimulation for Parkinson's disease. N Engl J Med 355(9):896-908, 2006

8. Doshi PK: Long-term surgical and hardware-related complications of deep brain stimulation. Stereotact Funct Neurosurg 89(2):89-95, 2011

9. Hariz Ml: Complications of deep brain stimulation surgery. Mov Disord 17 Suppl 3:S162-166, 2002

10. Hariz Ml: Safety and risk of microelectrode recording in surgery for movement disorders. Stereotact Funct Neurosurg 78(3-4):146-157, 2002

11. Janssen ML, Duits AA, Tourai AM, Ackermans L, Leentjes AF, van Kranen-Mastenbroek V, Oosterloo M, VisserVandewalle V, Temel Y: Subthalamic nucleus high-frequency stimulation for advanced Parkinson's disease: Motor and neuropsychological outcome after 10 years. Stereotact Funct Neurosurg 92(6):381-387, 2014

12. Kinfe TM, Vesper J: The impact of multichannel microelectrode recording (MER) in deep brain stimulation of the basal ganglia. Stereotact. Acta Neurochir Suppl 117:27-33, 2013

13. Kleiner-Fisman G, Herzog J, Fisman DN, Tamma F, Lyons KE, Pahwa R, Lang AE, Deuschl G: Subthalamic nucleus deep brain stimulation: Summary and meta-analysis of outcomes. Mov Disord 21 Suppl 14:S290-304, 2006

14. Kocabicak E, Alptekin O, Ackermans L, Kubben P, Kuijf M, Kurt E, Esselink R, Temel Y: Is there still need for microelectrode recording now the subthalamic nucleus can be well visualized with high field and ultrahigh MR imaging? Front Integr Neurosci 9:46, 2015

15. Kocabicak E, Aygun D, Ozaydin I, Jahanshahi A, Tan S, Onar M, Boke O, Kurt M, Guz H, Terzi M, Alptekin O, Temel Y: Does probe's eye subthalamic nucleus length on T2W MRI correspond with microelectrode recording in patients with deep brain stimulation for advanced parkinson's disease? Turk Neurosurg 23(5):658-665, 2013

16. Lyons KE, Wilkinson SB, Overman J, Pahwa R: Surgical and hardware complications of subthalamic stimulation A series of 160 procedures. Neurology 63(4):612-616, 2004

17. McClelland S: A cost analysis of intraoperative microelectrode recording during subthalamic stimulation for Parkinson's disease. Mov Disord 26(8):1422-1427, 2011

18. Odekerken VJ, van Laar T, Staal MJ, Mosch A, Hoffmann CF, Nijssen PC, Beute GN, van Vugt JP, Lenders MW, Contarino MF: Subthalamic nucleus versus globus pallidus bilateral deep brain stimulation for advanced Parkinson's disease (NSTAPS study): A randomised controlled trial. Lancet Neurology 12(1):37-44, 2013
19. Reck C, Maarouf M, Wojtecki L, Groiss SJ, Florin E, Sturm V, Fink GR, Schnitzler A, Timmermann L: Clinical outcome of subthalamic stimulation in Parkinson's disease is improved by intraoperative multiple trajectories microelectrode recording. J Neurol Surg A Cent Eur Neurosurg 73(6):377-386, 2012

20. Savas A, Bozkurt M, Akbostancı C: A comparison between stereotactic targeting methods of the subthalamic nucleus in cases with Parkinson's disease. Acta Neurochir Suppl 117:3541,2013

21. Schlaier JR, Habermeyer C, Warnat J, Lange M, Janzen A, Hochreiter A, Proescholdt M, Brawanski A, Fellner C: Discrepancies between the MRI-and the electrophysiologically defined subthalamic nucleus. Acta Neurochir (Wien) 153(12):2307-2318, 2011

22. Shamir RR, Zaidel A, Joskowicz L, Bergman H, Israel Z: Microelectrode recording duration and spatial density constraints for automatic targeting of the subthalamic nucleus. Stereotact Funct Neurosurg 90(5):325-334, 2012

23. Starr PA: Placement of deep brain stimulators into the subthalamic nucleus or globus pallidus internus: Technical approach. Stereotact Funct Neurosurg 79(3-4):118-145, 2002

24. Temel Y, Wilbrink P, Duits A, Boon P, Tromp S, Ackermans L, van Kranen-Mastenbroek V, Weber W, Visser-Vandewalle V: Single electrode and multiple electrode guided electrical stimulation of the subthalamic nucleus in advanced Parkinson's disease. Operative Neurosurgery 61 Suppl 5:ONS346-ONS57, 2007

25. Toda H, Sawamoto N, Hanakawa T, Saiki H, Matsumoto $\mathrm{S}$, Okumura $\mathrm{R}$, Ishikawa $\mathrm{M}$, Fukuyama $\mathrm{H}$, Hashimoto $\mathrm{N}$ : $\mathrm{A}$ novel composite targeting method using high-field magnetic resonance imaging for subthalamic nucleus deep brain stimulation. J Neurosurg 111(4):737-745, 2009

26. Tonge $M$, Ackermans $L$, Kocabicak $E$, van KranenMastenbroek V, Kuijf M, Oosterloo M, Kubben P, Temel Y: A detailed analysis of intracerebral hemorrhages in DBS surgeries. Clin Neurol Neurosurg 139:183-187, 2015

27. Tonge $M$, Kocabicak E, Ackermans L, Kuijf M, Temel Y: Final electrode position in subthalamic nucleus deep brain stimulation surgery: A comparison of indirect and direct targeting methods. Turk Neurosurg 26(6):900-903, 2016

28. Voges J, Waerzeggers $\mathrm{Y}$, Maarouf M, Lehrke R, Koulousakis A, Lenartz D, Sturm V: Deep-brain stimulation: Long-term analysis of complications caused by hardware and surgeryexperiences from a single centre. J Neurol Neurosurg Psychiatry 77(7):868-872, 2006

29. Xiaowu H, Xiufeng J, Xiaoping Z, Bin H, Laixing W, Yiqun C, Jinchuan L, Aiguo J, Jianmin L: Risks of intracranial hemorrhage in patients with Parkinson's disease receiving deep brain stimulation and ablation. Parkinsonism Relat Disord 16(2):96-100, 2010

30. Zeiler F, Wilkinson M, Krcek J: Subthalamic nucleus deep brain stimulation: An invaluable role for MER. Can J Neurol Sci 40(4):572-575, 2013

31. Zhu X, Hamel W, Schrader B, Weinert D, Hedderich J, Herzog J, Volkmann J, Deuschl G, Müller D, Mehdorn H: Magnetic resonance imaging-based morphometry and landmark correlation of basal ganglia nuclei. Acta Neurochir (Wien) 144(10):959-969, 2002 